\title{
Distribution of pseudo-critical temperatures and lack of self-averaging in disordered Poland-Scheraga models with different loop exponents
}

\author{
Cécile Monthus and Thomas Garel \\ Service de Physique Théorique, CEA/DSM/SPhT \\ Unité de recherche associée au CNRS \\ 91191 Gif-sur-Yvette cedex, France
}

\begin{abstract}
According to recent progresses in the finite size scaling theory of disordered systems, thermodynamic observables are not self-averaging at critical points when the disorder is relevant in the Harris criterion sense. This lack of self-averageness at criticality is directly related to the distribution of pseudo-critical temperatures $T_{c}(i, L)$ over the ensemble of samples $(i)$ of size $L$. In this paper, we apply this analysis to disordered Poland-Scheraga models with different loop exponents $c$, corresponding to marginal and relevant disorder. In all cases, we numerically obtain a Gaussian histogram of pseudo-critical temperatures $T_{c}(i, L)$ with mean $T_{c}^{a v}(L)$ and width $\Delta T_{c}(L)$. For the marginal case $c=1.5$ corresponding to two-dimensional wetting, both the width $\Delta T_{c}(L)$ and the shift $\left[T_{c}(\infty)-T_{c}^{a v}(L)\right]$ decay as $L^{-1 / 2}$, so the exponent is unchanged $\left(\nu_{\text {random }}=2=\nu_{\text {pure }}\right)$ but disorder is relevant and leads to non self-averaging at criticality. For relevant disorder $c=1.75$, the width $\Delta T_{c}(L)$ and the shift $\left[T_{c}(\infty)-T_{c}^{a v}(L)\right]$ decay with the same new exponent $L^{-1 / \nu_{\text {random }}}$ (where $\nu_{\text {random }} \sim 2.7>2>\nu_{\text {pure }}$ ) and there is again no self-averaging at criticality. Finally for the value $c=2.15$, of interest in the context of DNA denaturation, the transition is first-order in the pure case. In the presence of disorder, the width $\Delta T_{c}(L) \sim L^{-1 / 2}$ dominates over the shift $\left[T_{c}(\infty)-T_{c}^{a v}(L)\right] \sim L^{-1}$, i.e. there are two correlation length exponents $\nu=2$ and $\tilde{\nu}=1$ that govern respectively the averaged/typical loop distribution.
\end{abstract}

\section{INTRODUCTION}

The stability of pure critical points with respect to weak bond disorder is governed by the Harris criterion [1] : near a second order phase transition in dimension $d$, the bond disorder is irrelevant if the specific heat exponent is negative $\alpha_{\text {pure }}=2-d \nu_{\text {pure }}<0$ or equivalently if the correlation length exponent $\nu_{P} \equiv \nu_{\text {pure }}>2 / d$. On the contrary if $\nu_{P}<2 / d$, disorder is relevant and drives the system towards a random fixed point characterized by a new correlation length exponent satisfying the general bound $\nu_{R} \equiv \nu_{\text {random }} \geq 2 / d[2]$. More recently, important progresses have been made in the understanding of finite size properties of random critical points [3, 4, 5, 6, 7, 8]. The main outcome of these studies can be summarized as follows. To each disordered sample $(i)$ of size $L$, one should first associate a pseudo-critical temperature $T_{c}(i, L)$, defined for instance in magnetic systems as the temperature where the susceptibility is maximum $[3,5,6,6]$. The disorder averaged pseudo-critical critical temperature $T_{c}^{a v}(L) \equiv \overline{T_{c}(i, L)}$ satisfies

$$
T_{c}^{a v}(L)-T_{c}(\infty) \sim L^{-1 / \nu_{R}}
$$

where $\nu_{R}$ is the correlation length exponent. Eq. (11) generalizes the analogous relation for pure systems

$$
T_{c}^{\text {pure }}(L)-T_{c}(\infty) \sim L^{-1 / \nu_{P}}
$$

The nature of the disordered critical point then depends on the width $\Delta T_{c}(L)$ of the distribution of the pseudo-critical temperatures $T_{c}(i, L)$

$$
\Delta T_{c}(L) \equiv \sqrt{\operatorname{Var}\left[T_{c}(i, L)\right]}=\sqrt{\overline{T_{c}^{2}(i, L)}-\left(\overline{T_{c}(i, L)}\right)^{2}}
$$

When the disorder is irrelevant, the fluctuations of these pseudo-critical temperatures obey the scaling of a central limit theorem as in the Harris argument :

$$
\Delta T_{c}(L) \sim L^{-d / 2} \text { for irrelevant disorder }
$$

This behavior was first believed to hold in general [3, 5], but was later shown to be wrong in the case of random fixed points. In this case, it was argued [4, 6] that eq. (4) should be replaced by

$$
\Delta T_{c}(L) \sim L^{-1 / \nu_{R}} \quad \text { for random critical points }
$$


i.e. the scaling is the same as the $L$-dependent shift of the averaged pseudo-critical temperature (Eq. 11). The fact that these two temperature scales remain the same is then an essential property of random fixed points that leads to the lack of self-averaging for observables at criticality [4, 6]. More precisely, for a given observable $X$, it is convenient to define its normalized width as

$$
R_{X}(T, L) \equiv \frac{\overline{X_{i}^{2}(T, L)}-\left(\overline{X_{i}(T, L)}\right)^{2}}{\left(\overline{X_{i}(T, L)}\right)^{2}}
$$

If $\xi(T)$ denotes the correlation length, the following behavior of $R_{X}(T, L)$ is expected [4, 6]

(i) off criticality, when $L \gg \xi(T)$, the system can be divided into nearly independent sub-samples and this leads to 'Strong Self-Averaging'

$$
R_{X}(T, L) \sim \frac{1}{L^{d}} \quad \text { off criticality for } L \gg \xi(T)
$$

(ii) in the critical region, when $L \ll \xi(T)$, the system cannot be divided anymore into nearly independent subsamples. In particular at $T_{c}(\infty)$ where $\xi=\infty$, one can have either 'Weak Self-Averaging'

$$
R_{X}\left(T_{c}(\infty), L\right) \sim L^{\frac{\alpha_{P}}{\nu_{P}}} \quad \text { for irrelevant disorder }\left(\alpha_{P}<0\right)
$$

or 'No Self-Averaging'

$$
R_{X}\left(T_{c}(\infty), L\right) \sim C s t \quad \text { for random critical points }
$$

In this paper, we study from this point of view disordered Poland-Scheraga models with different loop exponents $c$, corresponding to either a pure second order transition with respectively marginal/relevant disorder according to the Harris criterion, or to a pure first-order transition. In each case, we numerically compute the histogram of pseudocritical temperatures and study the self-averaging properties at criticality. The paper is organized as follows. In Sec. III we recall the definition of Poland-Scheraga (PS) models, the critical properties of the pure transitions and the disorder relevance. In Sec. III we describe the observables that we numerically compute for disordered PS models. We then present our results for the different loop exponents considered, namely (i) $c=1.5$ (marginal disorder) in Sec. IV (ii) $c=1.75$ (relevant disorder) in Sec. V (iii) $c=2.15$ (case of a first-order transition in the pure case) in Sec VI.

\section{POLAND-SCHERAGA MODELS : DISORDER RELEVANCE AT PURE CRITICAL POINTS}

\section{A. Critical properties of pure Poland-Scheraga models}

We first consider the adsorption (or wetting) transition of a polymer chain onto an impenetrable substrate. This model corresponds to a Poland-Scheraga model with loop exponent $c=1.5$. This wetting model in dimension $1+1$ is defined as follows. The substrate is located at $z=0$. The polymer chain has $L$ monomers, and the position $z_{\alpha}$ of monomer $(\alpha)$ satisfies $z_{\alpha} \geq 0$, with $z_{1}=z_{L}=0$ (bound-bound boundary conditions). The partition function of the model reads

$$
Z=\sum_{(R W)} e^{-\beta H}
$$

where $H=\sum_{\alpha=1}^{L} \varepsilon_{\alpha} \delta_{z_{\alpha}, 0}$. In equation (10), $\beta=\frac{1}{k_{B} T}$ is the inverse temperature and the sum runs over all walks (RW) such that $\left|z_{\alpha+1}-z_{\alpha}\right|= \pm 1$. This problem can be formulated à la Poland-Scheraga [9] in terms of the loop statistics, where each loop of length $l$ has a weight $\frac{2^{l}}{l^{3 / 2}}$. We consider here generalized PS models where each loop of length $l$ has a weight $\frac{2^{l}}{l^{c}}$, with exponent $c>1$.

In the pure case $\varepsilon_{\alpha}=\varepsilon_{0}$, the loop distribution at $T_{c}=T_{c}(\infty)$ is

$$
P_{T c}^{\text {pure }}(l) \sim \frac{1}{l^{c}}
$$

For $c>2$, the averaged length $\left\langle l>=\int d l l P_{T c}^{\text {pure }}(l)\right.$ is finite, so that the number $n\left(T_{c}\right)$ of contacts with the substrate is extensive $\left(n\left(T_{c}\right) \sim L\right)$; the transition is therefore first order. For $1<c<2$, the averaged length $<>>$ diverges, 
and the Lévy sum of $n$ independent variables $l_{i}$ drawn from the distribution (11) scales as $l_{1}+\ldots+l_{n} \sim n^{1 /(c-1)}$. As a consequence at criticality, the number of contacts $n_{L}^{\text {pure }}\left(T_{c}\right)$ in a sample of length $L$ scales as

$$
n_{L}^{\text {pure }}\left(T_{c}\right) \sim L^{c-1}
$$

and the transition is second order. The contact density thus decays as

$$
\theta_{L}^{\text {pure }}\left(T_{c}\right)=\frac{n_{L}^{\text {pure }}\left(T_{c}\right)}{L} \sim L^{c-2}
$$

Its finite-size scaling form

$$
\theta_{L}^{\text {pure }}(T)=L^{c-2} Q\left[\left(T-T_{c}\right) L^{\frac{1}{\nu_{P}}}\right]
$$

involves the crossover exponent $\phi_{P}=\frac{1}{\nu_{P}}=c-1$.

\section{B. Harris criterion on $2 d$ order transitions $1<c<2$}

The Harris criterion [1] concerning the stability of pure second order transitions with respect to the addition of disorder relies on the sign of the specific heat exponent

$$
\alpha_{P}=2-\nu_{P}=\frac{2 c-3}{c-1}
$$

An equivalent way to decide whether disorder is relevant consists in a simple power-counting analysis of the disorder perturbation exactly at $T_{c}$ : the pure finite-size contact density $\left\langle\delta_{z_{i}, 0}>_{\text {pure }} \sim L^{c-2}\right.$ of Eq. (13) yields that the perturbation due to the presence of a small disorder in the contact energies $\epsilon_{i}=\epsilon_{0}+\delta \epsilon_{i}$ scales as

$$
\sum_{i=1}^{L} \delta \epsilon_{i}<\delta_{z_{i}, 0}>_{\text {pure }} \sim L^{1 / 2} \times L^{c-2}=L^{c-\frac{3}{2}}
$$

Disorder is thus irrelevant for $1<c<\frac{3}{2}$ and relevant for $\frac{3}{2}<c<2$. The marginal case $c=\frac{3}{2}$ has been debated for a long time 10, 11, 12, 13, 14, 15, 16] and is of special interest since it corresponds to two-dimensional wetting as explained above. As a consequence, we have chosen to study in parallel the cases $c=1.5$ and $c=1.75$.

\section{Disorder relevance on 1st order transitions $c>2$}

The effect of disorder on first-order transitions has been discussed for a long time [17, 18, 19, 20]. In this respect, the most frequently studied system [21, 22] is the 2D Potts model with $q>4$, for which the Aizenman-Wehr theorem 18] states that disorder changes the critical behavior from first order to second order. The recent numerical study of this phenomenon [22, 23] however shows that there are many subtleties : in particular, the latent heat that vanishes for continuous disorder, remains finite for binary disorder. Disorder effects on Potts models have also been studied in $3 \mathrm{D}[24]$.

Since all these studies consider spin systems displaying coexisting domains in the pure case, their conclusions cannot be directly applied to the PS-model for the following polymeric reasons [25] : in the PS model, there is no surface tension between the localized and delocalized phases, and there exists an diverging correlation length $1 /\left(T_{c}-T\right)$ in the pure case. So here, the simplest way to discuss the relevance of disorder consists in the simple power-counting analysis of the disorder perturbation exactly at $T_{c}$ as in Eq. (16). Here for $c>2$ where $\left\langle\delta_{z_{i}, 0}\right\rangle_{\text {pure }}$ is finite, the perturbation due to the presence of a small disorder in the contact energies $\epsilon_{i}=\epsilon_{0}+\delta \epsilon_{i}$ scales as

$$
\sum_{i=1}^{L} \delta \epsilon_{i}<\delta_{z_{i}, 0}>_{\text {pure }} \sim L^{1 / 2}
$$

As a consequence, disorder is relevant.

In this paper, we consider the case $c=2.15$ where the pure transition is first order, since this value is of interest for DNA denaturation [26, 27, 28, 29]. Furthermore, the effect of disorder on this transition has been recently debated [25, 30, 31]. 


\section{OBSERVABLES STUDIED IN DISORDERED PS MODELS}

\section{A. Numerical details}

As explained in details in our previous papers [16, 25], the PS models can be numerically studied via the recursion relations satisfied by the partition function, with a Fixman-Freire scheme [32] for the entropy of loops. All results presented in this paper have been obtained for independent quenched random contact energies $\left(\varepsilon_{\alpha}\right)$, distributed with a binary distribution. For $1<c<2$, we have chosen $\varepsilon=0$ or $\varepsilon=\varepsilon_{0}$ with probabilities $(1 / 2,1 / 2)$, with $\varepsilon_{0}(c=1.5)=-350 K$ and $\varepsilon_{0}(c=1.75)=-440 K$, to obtain critical temperatures in the same temperature range. For $c=2.15$ of interest in DNA denaturation, we have taken the same values as in our previous study [25], namely $\varepsilon=-355 K$ or $\varepsilon=-390 K$ with probabilities $(1 / 2,1 / 2)$.

The data we present correspond to various sizes $L$ with corresponding numbers $n_{s}(L)$ of independent samples. Unless otherwise stated, we have considered the following sizes going from $L=2 \cdot 10^{3}$ to $L=2048 \cdot 10^{3}$, with respectively $n_{s}\left(L=2 \cdot 10^{3}\right)=3.84 \cdot 10^{6}$ to $n_{s}\left(L=2048 \cdot 10^{3}\right)=15 \cdot 10^{3}$. More precisely, we consider

$$
\begin{aligned}
\frac{L}{1000} & =2,4,8,16,32,64,128,256,512,1024,2048 \\
\frac{n_{s}(L)}{1000} & =3840,1920,960,480,240,120,60,30,15,30,15
\end{aligned}
$$

\section{B. Definition of a sample-dependent pseudo-critical temperature}

In the magnetic systems studied in [3, 6], the pseudo-critical temperature $T_{c}(i, L)$ of the sample $i$ was identified to the maximum of the susceptibility. In the PS model, one can not follow the same path and we have tried two different definitions :

Definition of a pseudo-critical temperature from the free-energy

In the pure PS model with bound-bound boundary conditions, the behavior of the partition function as a function of temperature reads

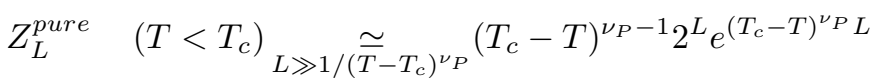

$$
\begin{aligned}
& Z_{L}^{\text {pure }} \quad\left(T_{c}\right) \simeq \frac{2^{L}}{L^{2-c}} \\
& Z_{L}^{\text {pure }} \quad\left(T>T_{c}\right) \underset{L \gg 1 /\left(\bar{T}-T_{c}\right)^{\nu_{P}}}{\simeq} \frac{2^{L}}{\left(T-T_{c}\right)^{2} L^{c}}
\end{aligned}
$$

with $\nu_{P}=1 /(c-1)$. A finite-size pseudo-critical temperature $T_{c}^{\text {pure }(f)}(L)$ can then be defined as the temperature where the free-energy reaches the extensive delocalized value $F_{\text {deloc }}=-T L \ln 2$, i.e. $T_{c}^{\text {pure }(f)}(L)$ is the solution of the equation

$$
F_{L}^{\text {pure }}(L, T)+T L \ln 2=0
$$

This definition from the free-energy is very natural, but has the drawback of introducing a logarithmic factor

$$
T_{c}^{\text {pure }(f)}(L)-T_{c}(\infty) \sim\left(\frac{\ln L}{L}\right)^{1 / \nu_{P}}
$$

with respect to the purely algebraic factor usually expected (Eq. 21). This logarithmic factor comes the finite-size free-energy value exactly at criticality $F_{L}^{\text {pure }}\left(L, T_{c}\right)=-T_{c} L \ln 2+(2-c) T_{c} \ln L$ (Eq. 20). In the disordered case, we may similarly define a sample-dependent pseudo-critical temperature $T_{c}^{(f)}(i, L)$ as the solution of the equation

$$
F_{L}^{(i)}(L, T)+T L \ln 2=0
$$

but logarithmic corrections are to be expected at least in the shift (Eq. 1). Since these logarithmic factors may alter the numerical measures of critical exponents in the disordered cases, we have also considered another definition of $T_{c}(i, L)$.

Definition of a pseudo-critical temperature from a sample-replication procedure 
In the pure PS model with $1<c<2$, another finite-size critical temperature $T_{c}^{\text {pure }(\theta)}(L)$ may be defined as the temperature where the appropriately rescaled contact densities cross (Eq. 13)

$$
L^{2-c} \theta_{L}^{\text {pure }}\left[T_{c}^{\text {pure }(\theta)}(L)\right]=(2 L)^{2-c} \theta_{2 L}^{\text {pure }}\left[T_{c}^{\text {pure }(\theta)}(2 L)\right]
$$

This temperature $T_{c}^{\text {pure }(\theta)}(L)$ follows the usual algebraic behavior (Eq. 2), in contrast with the other definition $T_{c}^{\text {pure }(f)}(L)$ that introduces logarithmic corrections (22).

This definition can be extended to the disordered case via the following strategy first introduced in [25] for the case $c=2.15$ (with bound-unbound boundary conditions ). In short, one considers a sample $(i)$ of length $L$, the sample (2i) of length $2 L$, obtained by gluing together two copies of sample $i$, as well as the sample (4i) obtained by gluing together four copies of sample $i$ : the three contact densities of $(i),(2 i)$ and (4i) cross at a temperature (see Fig. 9b of [25]) that can be interpreted as the pseudo-critical temperature $T_{c}(i, L)$ of the disordered sample $(i)$ of length $L$.

Here we generalize this procedure to the case $1<c<2$. The basic idea is that in disordered Poland-Scheraga models, the loop distribution has exponent $c$ at criticality in the thermodynamic limit as in the pure case (11). We have found numerically this property in our previous studies on the wetting transition with $c=1.5$ [16] and on the DNA denaturation with $c=2.15$ [25]. This suggests that the density of contacts scales as $L^{2-c}$ at criticality as in (13).

For $1<c<2$, we have thus tried to define a sample-dependent pseudo-critical temperature $T_{c}(i, L)$ as follows. For each sample $(i)$ of length $L$, we construct the sample $(2 i)$ of length $2 L$ by gluing together two copies of sample $i$, as well as the sample (4i) by gluing together four copies of sample $i$. We then plot the rescaled contact densities

$$
y_{T}(i, L)=\theta_{T}(i, L) L^{2-c}
$$

with its two analogs $y_{T}(2 i, 2 L)=\theta_{T}(2 i, 2 L)(2 L)^{2-c}$ and $y_{T}(4 i, 4 L)=\theta_{T}(4 i, 4 L)(4 L)^{2-c}$. Typical results obtained respectively for the cases $c=1.5$ and $c=1.75$ with two disordered samples $i=i_{1}, i_{2}$ are shown on Fig. 1]: for each sample $(i)$, the three rescaled contact densities $y_{T}(i, L), y_{T}(2 i, 2 L)$ and $y_{T}(4 i, 4 L)$ cross at a temperature that we defined as the pseudo-critical temperature $T_{c}^{(\theta)}(i, L)$ of the disordered sample $i$ of length $L$. This crossing of three curves validates the present replication procedure to define a proper pseudo-critical temperature.
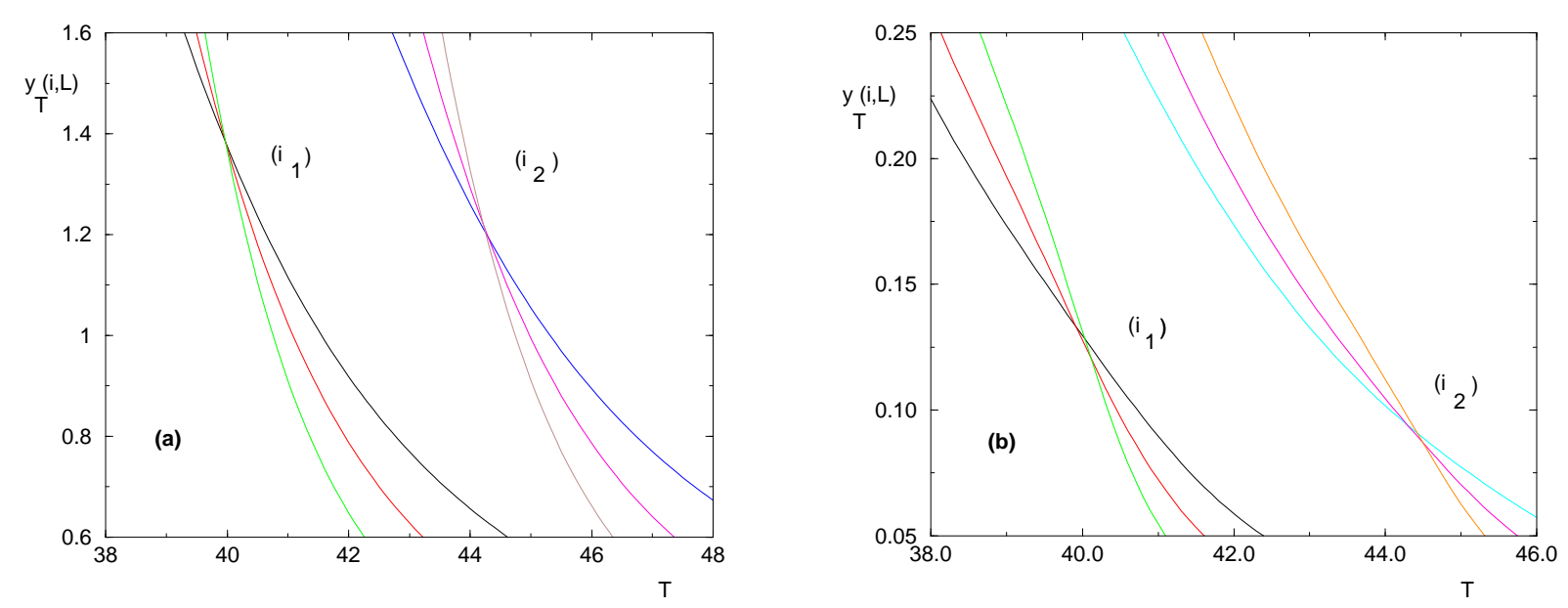

FIG. 1: Illustration of the crossing procedure to obtain the pseudo-critical temperatures $T_{c}(i, L)$ for two samples $i=i_{1}, i_{2}($ a) for the case $\mathrm{c}=1.5$ with $\mathrm{L}=50000$ (b) for the case $c=1.75$ with $L=100000$.

\section{Discussion}

Each definition of $T_{c}(i, L)$ has its own advantage and drawback. The definition $T_{c}^{(f)}(i, L)$, that uses the free-energy, is probably the most natural one, but introduces logarithmic corrections, already in the pure PS model. The definition $T_{c}^{(\theta)}(i, L)$, that uses the sample-replication procedure, may appear more artificial in the disordered case, but it does not introduce logarithmic corrections. We have checked numerically that both definitions actually yield very similar results for critical exponents and scaling distributions, even though they give different values for a given sample. This shows that the conclusions that can be obtained do not depend on the precise definition of the pseudo-critical temperature. 


\section{Distribution of pseudo-critical temperatures}

In all cases $(c=1.5,1.75$ and 2.15), and for both definitions of the pseudo-critical temperature (either from the free-energy or from the sample-replication procedure), we numerically obtain that the distributions of pseudo-critical temperatures follows the scaling form

$$
P_{L}\left(T_{c}(i, L)\right) \simeq \frac{1}{\Delta T_{c}(L)} g\left(x=\frac{T_{c}(i, L)-T_{c}^{a v}(L)}{\Delta T_{c}(L)}\right)
$$

where the scaling distribution $g(x)$ is simply Gaussian

$$
g(x)=\frac{1}{\sqrt{2 \pi}} e^{-x^{2} / 2}
$$

This means that the only important properties of the pseudo-critical temperatures distribution are the behaviors of its average $T_{c}^{a v}(L)$ and width $\Delta T_{c}(L)$ as $L$ varies.

For the average $T_{c}^{a v}(L)$, we have fitted our data with the power-law (Eq 1 for the temperature $T_{c}^{(\theta)}(i, L)$, and with the generalized form involving logarithm (see Eq. 22 for the pure case) for the temperature $T_{c}^{(f)}(i, L)$

$$
\begin{aligned}
& T_{c}^{a v(\theta)}(L)-T_{c}(\infty) \simeq-A_{\theta}\left(\frac{1}{L}\right)^{1 / \nu_{R}} \\
& T_{c}^{a v(f)}(L)-T_{c}(\infty) \simeq-A_{f}\left(\frac{\ln L}{L}\right)^{1 / \nu_{R}}
\end{aligned}
$$

The two definitions of $T_{c}(i, L)$ then yield compatible estimates for the exponent $\nu_{R}$ and the critical temperature $T_{c}(\infty)$.

For the variance, we have fitted our data with the power-law (Eq. 5. for both definitions of pseudo-critical temperatures, and the two definitions of $T_{c}(i, L)$ then yield compatible estimates for the corresponding exponent.

\section{Study of self-averageness in the critical region}

As already mentioned in the Introduction, the issue of self-averageness at random critical points is directly related to the scale of the width of the pseudo-critical temperatures [4, [6] : if the width $\Delta T_{c}(L)$ is of the same order of the shift $T_{c}^{a v}(L)-T_{c}(\infty)$, there is no self-averaging at $T_{c}(\infty)$.

We have thus measured for PS-models the $L$ dependence of the ratio $R_{\theta}(T, L)$ defined in Eq. (6) for the contact density for temperature $T$ in the critical region

$$
R_{\theta}(T, L) \equiv \frac{\operatorname{Var}\left[\theta_{i}(T, L)\right]}{\left(\overline{\theta_{i}(T, L)}\right)^{2}}
$$

We have also studied the issue of self-averageness at $T_{c}(i, L)$ via the measure of the ratio

$$
\mathcal{R}_{c}(L) \equiv \frac{\operatorname{Var}\left[\theta_{i}\left(T_{c}(i, L), L\right)\right]}{\left(\overline{\theta_{i}\left(T_{c}(i, L), L\right)}\right)^{2}}
$$

We now make contact with the finite size scaling Ansatz of [3], [6] for an observable $X$

$$
X_{L}^{(i)}(T)=L^{\rho} Q_{i}\left(\left(T-T_{c}(i, L)\right) L^{1 / \nu_{R}}\right)
$$

where sample dependence arises through both $T_{c}(i, L)$ and the scaling function $Q_{i}$. The ratio $R_{\theta}(T, L)$ mostly tests the relevance of the variance $\Delta T_{c}(L)$, whereas the ratio $\mathcal{R}_{c}(L)$ of Eq. (31) directly tests the sample dependence of $Q_{i}(0)$ for the observable $X=\theta$. We have measured the ratio $\mathcal{R}_{c}(L)$ for both definitions of $T_{c}(i, L)$. Although numerically different, their qualitative behaviour is the same: whenever disorder is relevant, the ratio $\mathcal{R}_{c}(L)$ grows with $L$.

We now present our results for the different loop exponents $c$. 


\section{THE CASE OF MARGINAL DISORDER $(c=1.5)$}

The marginal case $c=1.5$ corresponds to two-dimensional wetting and has been the subject of a long-standing debate [10, 11, 12, 13, 14, 15, 16]. On the analytical side, efforts have focused on the small disorder limit : Ref 10$]$ finds a marginally irrelevant disorder where the quenched critical properties are the same as in the pure case, up to subdominant logarithmic corrections. Other studies have concluded that that the disorder is marginally relevant [1], 12, 13]. On the numerical side, the same debate on the disorder relevance took place. The numerical studies of Ref. [10] and Ref. [14] have concluded that the critical behavior was indistinguishable from the pure transition. On the other hand, the numerical study of 11 ] pointed towards a negative specific heat exponent $(\alpha<0)$, and finally Ref. [15] has been interpreted as an essential singularity in the specific heat, that formally corresponds to an exponent $\alpha=-\infty$.

It is thus very interesting to study the marginal case $c=1.5$ from the point of view of the histogram of pseudo-critical temperatures and of self-averaging properties to clarify the situation.

\section{A. Distribution of pseudo-critical temperatures}

The histograms of both definitions of the pseudo critical temperatures $T_{c}(i, L)$ are shown on Figure 2(a) and (b). As mentioned previously, both rescaled distributions shown in the Insets of Figure 2] are Gaussian (Eq. 26).
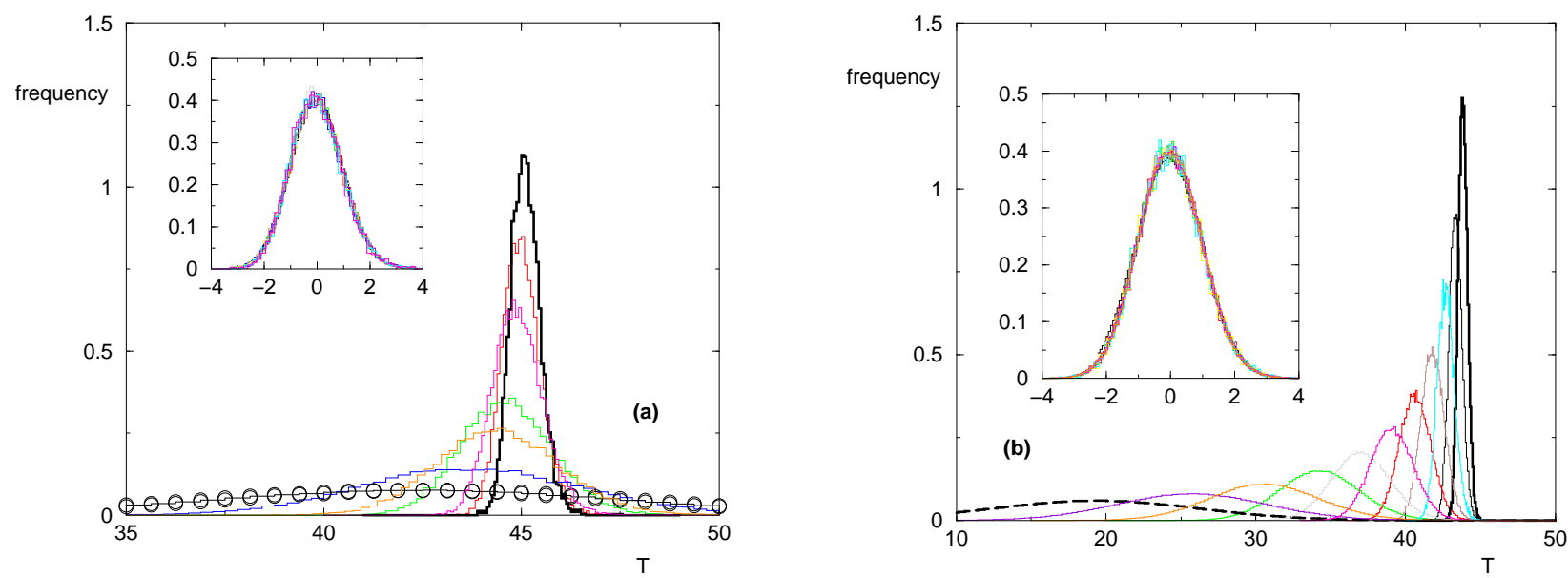

FIG. 2: Case $\mathrm{c}=1.5$ : Distribution of pseudo-critical temperatures $T_{c}(i, L)$, together with the rescaling of Eq. (26) in Inset. (a) for definition $T_{c}^{(\theta)}(i, L)$ with samples size from $L / 1000=4(\bigcirc)$ to 2048 (thick line). (b) for definition $T_{c}^{(f)}(i, L)$ with samples size from $L / 1000=2$ (dashed line) to 2048 (thick line)

The averages $T_{c}^{a v}(L)$ behave as

$$
\begin{aligned}
& T_{c}^{a v(\theta)}(L) \simeq 45.15-132\left(\frac{1}{L}\right)^{0.47} \\
& T_{c}^{a v(f)}(L) \simeq 45.25-335\left(\frac{\ln L}{L}\right)^{0.46}
\end{aligned}
$$

These values of $T_{c}(\infty) \sim 45.2$ agree with a different determination via the loop statistics in [16]. The mesaured exponent $\nu_{R}$ is very close to the pure exponent $\nu_{P}=2$. However, this does not mean that the disorder is irrelevant, since power-law fits of the variances yield

$$
\begin{aligned}
\Delta T_{c}^{(\theta)}(L) & \simeq 206\left(\frac{1}{L}\right)^{0.44} \\
\Delta T_{c}^{(f)}(L) & \simeq 188\left(\frac{1}{L}\right)^{0.44}
\end{aligned}
$$

Note that disorder being marginal, extra logarithmic factors could be present in the shift and in the variance. Theoretical predictions on this point are however unavailable, and we have not tried to include these extra factors in the above fits. 
Our conclusion is that the shift and the width have very close exponents, pointing towards a random critical point (Eq. 5) with non self-averaging properties that we now consider.

\section{B. Non-self-averaging properties}

We show on Figure 3 (a) the behavior of the ratio $R_{\theta}(T, L)$ defined in (Eq. 30). Near the critical temperature $T_{c}(\infty) \sim 45.2$ obtained in Eq 33 this ratio clearly displays a lack of self averaging, as expected at random critical points (Eq. 9).

We show on Figure 3 (b) the ratios $\mathcal{R}_{c}(L)$ of Eq. (31) corresponding to the two definitions of $T_{c}(i, L)$. They both grow with $L$.
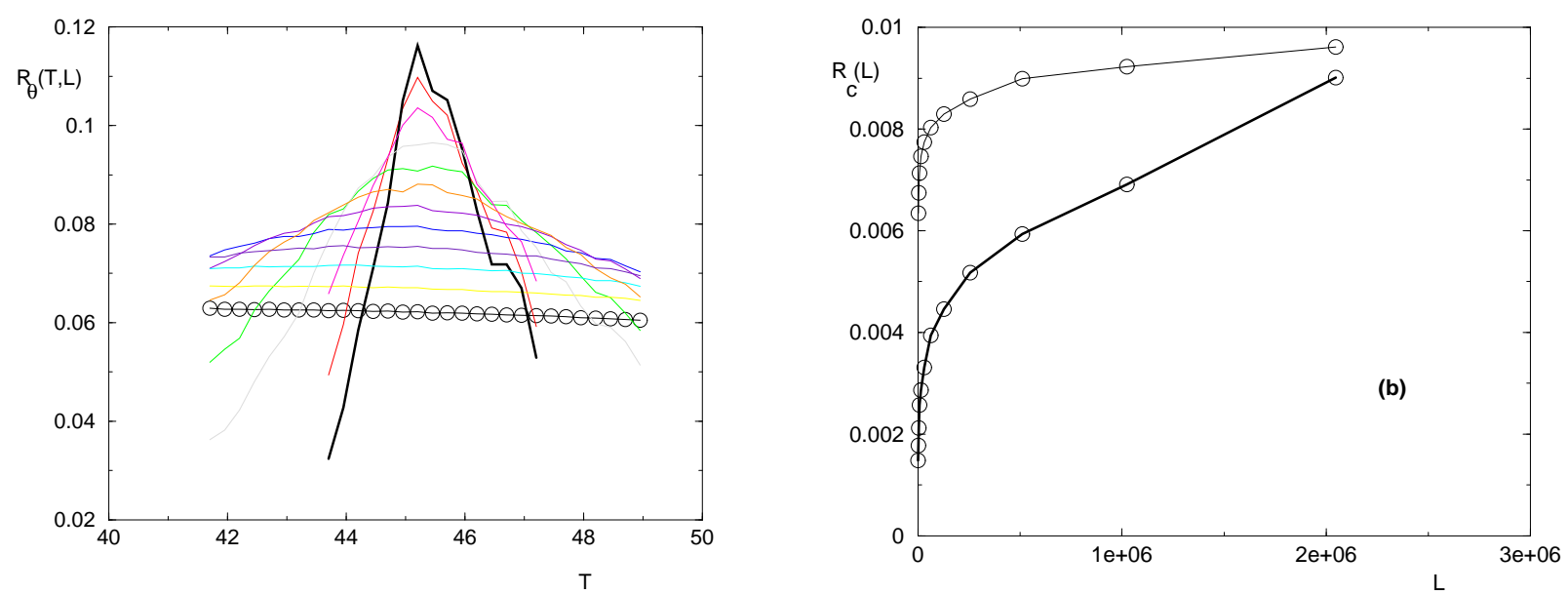

FIG. 3: Case c=1.5 : (a) Ratio $R_{\theta}(T, L)$ for sizes $L / 1000=1(\bigcirc)$ to 2048 (thick line) (b) The ratios $\mathcal{R}_{c}(L)$ of $($ Eq. 31), corresponding to definitions $T_{c}^{(\theta)}(i, L)$ (thick line) and $T_{c}^{(f)}(i, L)$ (thin line), both grow with $L$.

\section{Conclusion on the nature of the transition for $c=1.5$}

Our numerical results (33 34 indicate that in the presence of disorder, the shift- and width- exponents are close to each other and to the pure case exponent $\frac{1}{\nu_{\text {pure }}}=0.5$. We thus conclude

$$
\nu_{\text {random }}=2=\nu_{\text {pure }}
$$

The disorder is nevertheless relevant in the sense that there is a lack of self-averaging properties at criticality as in Eq. (9), because both the width $\Delta T_{c}(L)$ and the shift $\left[T_{c}(\infty)-T_{c}^{a v}(L)\right]$ decay with the same exponent

$$
\Delta T_{c}(L) \sim\left[T_{c}(\infty)-T_{c}^{a v}(L)\right] \sim L^{-1 / 2}
$$

\section{THE CASE OF RELEVANT DISORDER $(c=1.75)$}

\section{A. Distribution of pseudo-critical temperatures}

The histograms of both definitions of the pseudo critical temperatures $T_{c}(i, L)$ are shown on Figure 4 (a) and (b). Both rescaled distributions shown in the Insets of Figure 4 are Gaussian (Eq. 26).

The respective averaged $T_{c}^{a v}(L)$ behave as

$$
\begin{aligned}
& T_{c}^{a v(\theta)}(L) \simeq 45.2-155\left(\frac{1}{L}\right)^{0.35} \\
& T_{c}^{a v(f)}(L) \simeq 45.4-140\left(\frac{\ln L}{L}\right)^{0.37}
\end{aligned}
$$



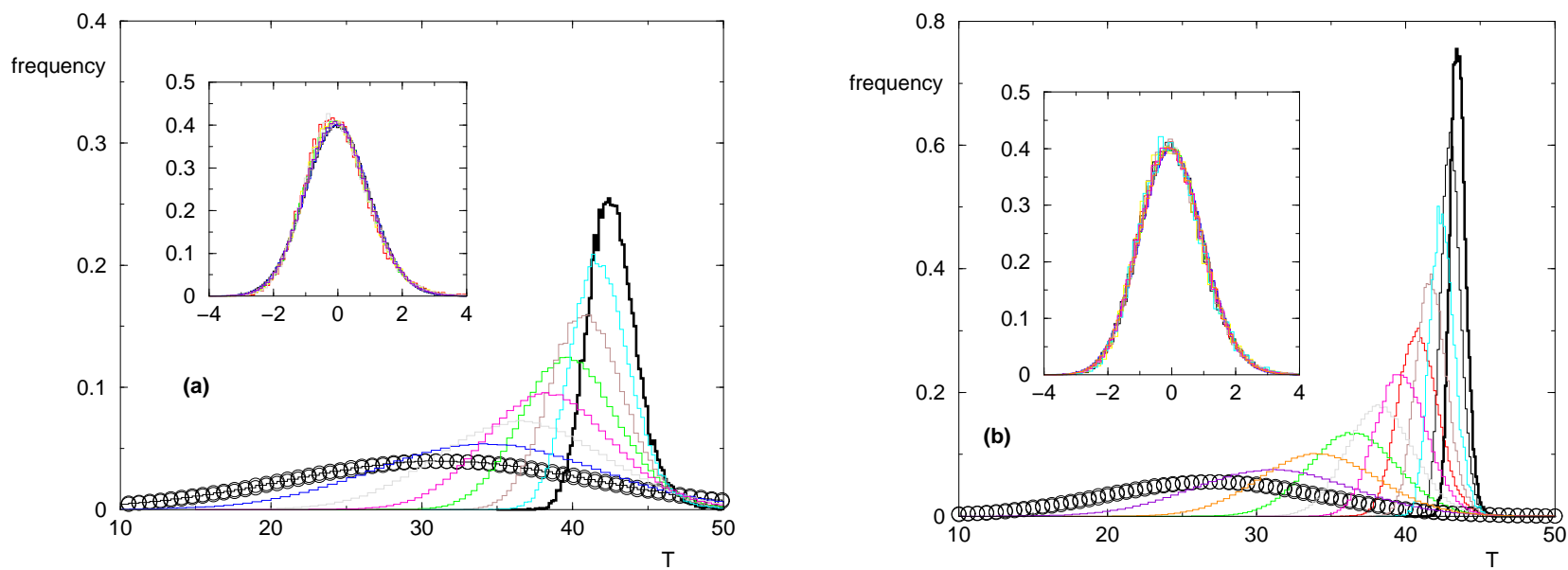

FIG. 4: Case $\mathrm{c}=1.75$ : Distribution of pseudo-critical temperatures $T_{c}(i, L)$, together with the rescaling of Eq. (26) in Inset. (a) for definition $T_{c}^{(\theta)}(i, L)$ with samples size from $L / 1000=1(\bigcirc)$ to 128 (thick line). (b) for definition $T_{c}^{(f)}(i, L)$ with samples size from $L / 1000=2(\bigcirc)$ to 2048 (thick line)

This shift with the size $L$ shows (Eq. 11) that the exponent $1 / \nu_{R}=0.35$ is very different from the pure exponent $1 / \nu_{P}=c-1=0.75$. As expected, the random exponent satisfies the general bound $\nu_{R} \geq 2$ [2]. For both definitions the widths decay with the same exponent, namely

$$
\begin{aligned}
& \Delta T_{c}^{(\theta)}(L) \simeq 134\left(\frac{1}{L}\right)^{0.38} \\
& \Delta T_{c}^{(f)}(L) \simeq 117\left(\frac{1}{L}\right)^{0.38}
\end{aligned}
$$

in agreement with the prediction of Eq. (5)

$$
\Delta T_{c}(L) \sim T_{c}^{a v}(L)-T_{c}(\infty)
$$

for random critical points.

\section{B. Non-self-averaging properties}

We show on Figure[5 (a) the behavior of the ratio $R_{\theta}(T, L)$ defined in (30). It clearly displays a lack of self averaging, as expected at random critical points (Eq. 9) .

We show on Figure 5 (b) the ratios $\mathcal{R}_{c}(L)$ of Eq. (31) corresponding to the two definitions of $T_{c}(i, L)$. They both grow with $L$.

\section{Conclusion on the transition for $c=1.75$}

For relevant disorder $c=1.75$, our conclusion is that the width $\Delta T_{c}(L)$ and the shift $\left[T_{c}(\infty)-T_{c}^{a v}(L)\right]$ decay with the same new exponent $L^{-1 / \nu_{\text {random }}}$ (where $\nu_{\text {random }} \sim 2.7>2>\nu_{\text {pure }}$ ) and there is no self-averaging at criticality, in agreement with the general predictions (5) and (9) for random critical points.

\section{DISORDER EFFECTS ON A FIRST ORDER TRANSITION $(c=2.15)$}

The case $c=2.15$ is of special interest in the context of DNA denaturation, and the nature of the transition in the presence of disorder has been under debate recently [25, 30, 31]. The numerical studies [25, 30] with bound-unbound boundary conditions have found crossings of the energy density (Fig. 4 of [30] and Fig. 7b of [25]) and of the contact density (Fig. 6 (b) of [25]) for various sizes $L$. These results point towards a finite energy density and a finite contact 

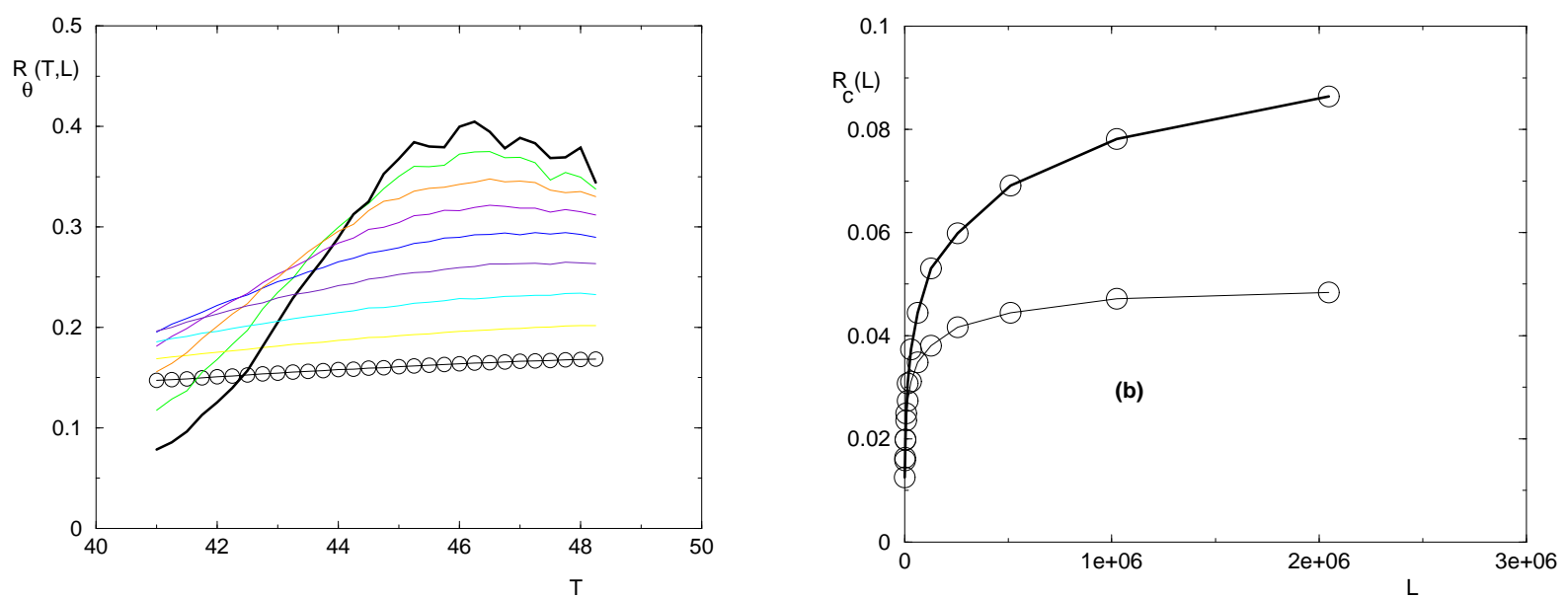

FIG. 5: Case c=1.75 : (a) Ratio $R_{\theta}(T, L)$ for sizes $L / 1000=1(\bigcirc), 2,4,8,16,32,64,128,256$ (thick line) (b) The ratios $\mathcal{R}_{c}(L)$ of (Eq. 31), corresponding to definitions $T_{c}^{(\theta)}(i, L)$ (thick line) and $T_{c}^{(f)}(i, L)$ (thin line), both grow with $L$.

density at criticality. However, a recent probabilistic analysis [31] of the disordered PS model with $c>2$ has concluded that the second derivative of the free-energy remains bounded when approaching the critical point from the localized phase, and that the order parameter vanishes continuously. It is thus interesting to reconsider the problem from the point of view of the distribution of pseudo-critical temperatures to clarify the nature of the transition.

\section{A. Distribution of pseudo-critical temperatures}

The histograms of both definitions of the pseudo critical temperatures $T_{c}(i, L)$ are shown on Figure 6(a) and (b). Both rescaled distributions shown in the Insets of Figure 6 are Gaussian (Eq. 26).
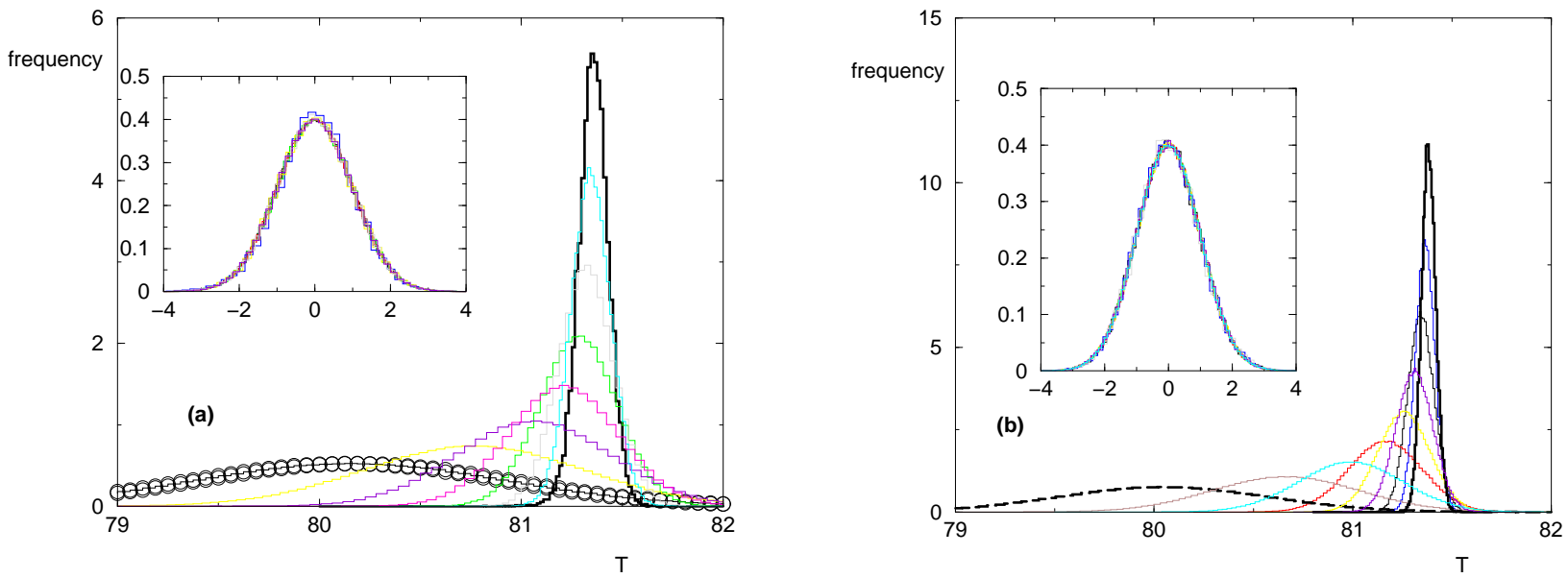

FIG. 6: Case $\mathrm{c}=2.15$ : Distribution of pseudo-critical temperatures $T_{c}(i, L)$, together with the rescaling of Eq. (26) in Inset. (a) for definition $T_{c}^{(\theta)}(i, L)$ with samples size from $L / 1000=1(\bigcirc)$ to 128 (thick line) (b) for definition $T_{c}^{(f)}(i, L)$ with samples size from $L / 1000=2$ (dashed line) to 512 (thick line)

The averages $T_{c}^{a v}(L)$ behave as

$$
\begin{aligned}
T_{c}^{a v(\theta)}(L) & \simeq 81.36-\frac{1254}{L} \\
T_{c}^{a v(f)}(L) & \simeq 81.38-357\left(\frac{\ln L}{L}\right)
\end{aligned}
$$

The two values of $T_{c}(\infty)$ are close and agree with the value obtained in Ref. [25] with a different method. On one 
hand, according to Eq. 11 this behavior of the shift seems to indicate that the exponent is unchanged with respect to the pure case $\nu_{R}=1=\nu_{P}$. On the other hand, the width is found to be much bigger than the shift since

$$
\begin{aligned}
\Delta T_{c}^{(\theta)}(L) & \simeq 23.8\left(\frac{1}{L}\right)^{0.49} \\
\Delta T_{c}^{(f)}(L) & \simeq 23.2\left(\frac{1}{L}\right)^{0.49}
\end{aligned}
$$

This behavior corresponds to the Central Limit estimation $L^{-1 / 2}$, as in the Harris argument.

The fact that the shift and the width exhibit different exponents actually means that two diverging correlation lengths coexist in the presence of disorder

$$
\begin{aligned}
\xi_{\text {shift }}(T) & \sim \frac{1}{\left(T_{c}-T\right)} \\
\xi_{\text {var }}(T) & \sim \frac{1}{\left(T_{c}-T\right)^{2}}
\end{aligned}
$$

The presence of two different correlation length exponents was already found in other models, in particular in the random transverse field Ising chain [33], where the exponent $\nu=2$ governs the decay of averaged correlation, whereas $\tilde{\nu}=1$ governs the decay of typical correlations. In Sec. VII A of Ref. [33], a scaling analysis of disorder effects on first-order phase transitions in dimension $d$ suggests that the presence of two different correlation length exponents $\nu=2 / d$ and $\tilde{\nu}=1 / d$ should be generic in these systems : the exponent $\tilde{\nu}=1 / d$ is expected to describe the rounding of the transition in a typical sample, whereas $\nu=2 / d$ describes the rounding of the transition of the distribution of samples. We will discuss this issue in more details in Section VID after the description of our numerical results on non-self-averaging properties.

\section{B. Non-self-averaging properties}

We show on Figure[7 (a) the behavior of the ratio $R_{\theta}(T, L)$ defined in (30). It clearly displays a lack of self averaging at criticality.

We show on Figure 7 (b) the ratios $\mathcal{R}_{c}(L)$ of Eq. (31) corresponding to the two definitions of $T_{c}(i, L)$. They both grow with $L$.
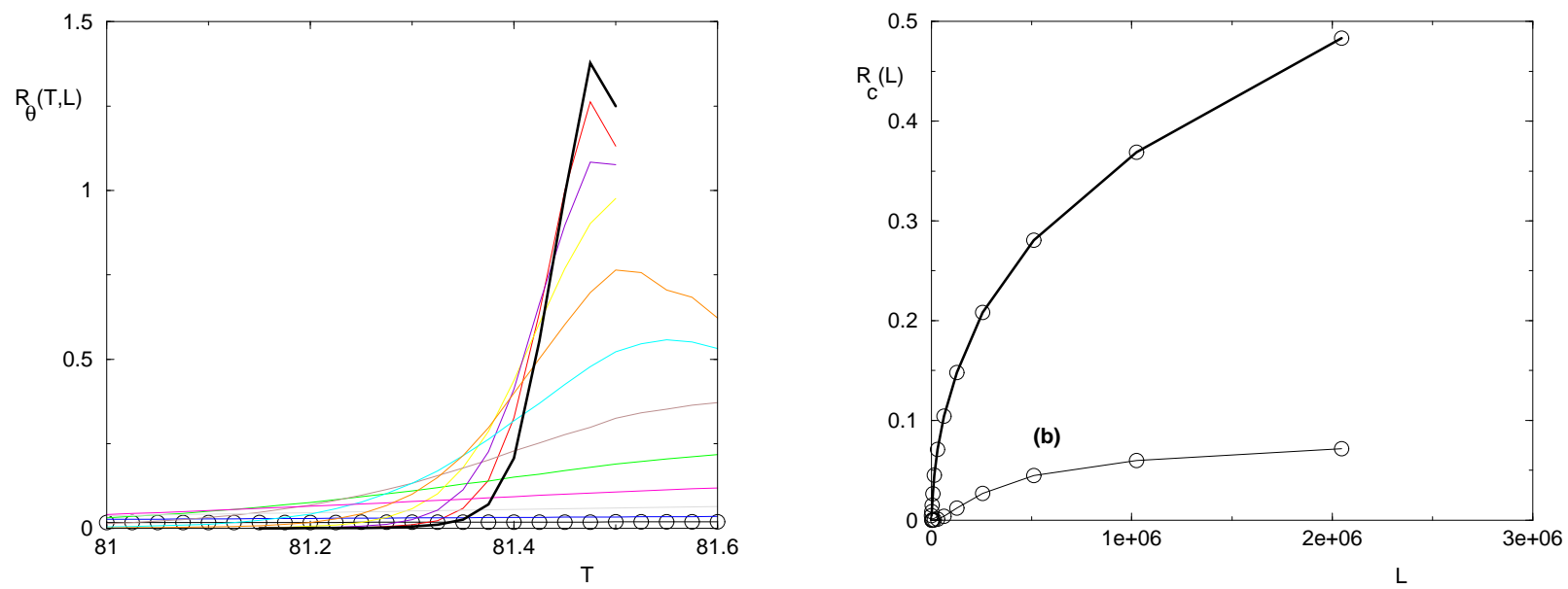

FIG. 7: Case c=2.15 : (a) Ratio $R_{\theta}(T, L)$ for sizes $L / 1000=1(\bigcirc)$ to 2048 (thick line) (b) The ratios $\mathcal{R}_{c}(L)$ of $($ Eq. 31), corresponding to definitions $T_{c}^{(\theta)}(i, L)$ (thick line) and $T_{c}^{(f)}(i, L)$ (thin line), both grow with $L$. 


\section{Finiteness of the contact density at criticality}

The dominance of the variance (41) over the shift (40) indicate that asymptotically for large $L$, half of the samples $(i, L)$ are still localized at $T_{c}(\infty)$, whereas the other half is already delocalized. This suggests that the contact density is finite at criticality, as we have numerically found in our previous study 25]. However, since Ref. 31] states that the order parameter vanishes continuously at the transition, we have performed more detailed calculations. Figure 8 (which is more detailed than Figure 6 (b) of our previous study [25] ) shows the averaged contact density $\overline{\theta_{L}(T)}$ as a function of the temperature $T$ for several sizes : the results cross regularly without rescaling. This points towards a finite contact density at criticality.

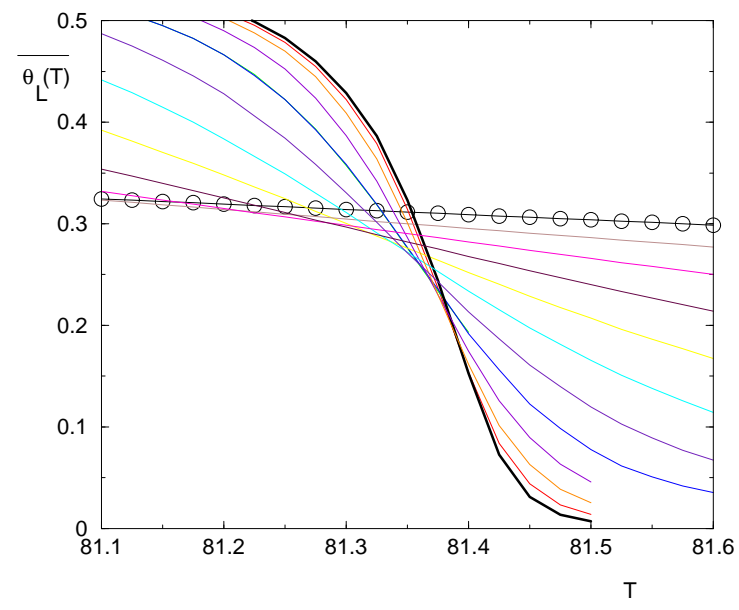

FIG. 8: Case $c=2.15$ : crossing of the averaged contact density $\overline{\theta_{L}(T)}$ for sizes from $L / 1000=1(\bigcirc)$ to 2048 (thick line) .

\section{Discussion on the nature of the transition for $c=2.15$ in the presence of disorder}

Our present study shows that, in the presence of disorder, the transition for $c=2.15$ is an unconventional random critical point with two different correlation length exponents $\nu=2$ and $\tilde{\nu}=1$ (42). This is in contrast with usual random critical points, arising from second order transitions with relevant disorder, where the same exponent is expected to govern the width and the shift (Eq. [5), but this is reminiscent of what happens at strong disorder fixed points [33, 34]. The question is now which correlation exponent appears in a given observable. In the random transverse field Ising chain where many exacts results are known for exponents and scaling distribution functions [33], it is well understood how the two exponents $\nu=2$ and $\tilde{\nu}=1$ govern respectively the averaged/typical correlations. Here in the disordered PS model, the analog of the correlation function is the loop distribution. To simplify the discussion, let us more specifically consider the probability of an end-to-end loop of length $L$ in sample (i) of length $L$, which is directly related to the partition function $Z_{L}^{(i)}(T)$ of sample $(i)$

$$
P_{L}^{(i)}(L, T)=\frac{2^{L}}{L^{c}} \frac{1}{Z_{L}^{(i)}(T)}
$$

Introducing for each sample $(i)$ the difference between the free-energy density $F_{L}^{(i)}(T) / L=-T \ln Z^{(i)}(L, T) / L$ and the delocalized value $f_{\text {deloc }}=-T \ln 2$

$$
f^{(i)}(L, T) \equiv \frac{-T \ln Z^{(i)}(L, T)}{L}+T \ln 2
$$

one gets

$$
\ln P_{L}^{(i)}(L, T)=-c \ln L+L \beta f^{(i)}(L, T)
$$

The self-averaging property of the free energy means that $f_{L}^{(i)}(T)$ converge for large $L$ to a non-random value $f(T)$ for any sample $(i)$ with probability one

$$
f_{L}^{(i)}(T) \underset{L \rightarrow \infty}{\longrightarrow} f(T)
$$


where $f(T)$ is the free-energy difference between the localized phase and the delocalized phase $: f\left(T<T_{c}\right)<0$ and $f\left(T>T_{c}\right)=0$. This translates immediately into the corresponding statement (45) for the logarithm of end-to-end loop probability

$$
\frac{\ln P_{L}^{(i)}(L, T)}{L} \underset{L \rightarrow \infty}{\longrightarrow} \beta f(T)
$$

for any sample $(i)$ with probability one. Since the typical correlation length $\tilde{\xi}(T)$ is usually defined as the decay rate of the logarithm of the correlation, we obtain here that it is simply given by the inverse of the free-energy $f(T)$

$$
\frac{1}{\tilde{\xi}(T)} \equiv-\lim _{L \rightarrow \infty}\left(\frac{\ln P_{L}^{(i)}(L, T)}{L}\right)=-\beta f(T)
$$

Here for $c=2.15$, we expect from the discussion of the previous Section VIC that the contact density is finite at criticality : this implies that the energy is also finite at criticality, and thus we are led to the conclusion that the free-energy vanishes linearly

$$
f(T) \underset{T \rightarrow T_{c}^{-}}{\longrightarrow}\left(T_{c}-T\right)
$$

The typical correlation length involves the exponent $\tilde{\nu}=1$

$$
\tilde{\xi}(T) \underset{T \rightarrow T_{c}^{-}}{\longrightarrow}\left(T_{c}-T\right)^{-\tilde{\nu}} \text { with } \tilde{\nu}=1
$$

Let us now consider the decay of the averaged end-to-end loop distribution that defines an a priori different correlation length $\xi(T)$

$$
\frac{\ln \left(\overline{P_{L}^{(i)}(L, T)}\right)}{L} \underset{L \rightarrow \infty}{\longrightarrow}-\frac{1}{\xi(T)}
$$

This correlation length $\xi(T)$ determines the divergence of high moments of the averaged loop distribution. At a given temperature $T<T_{c}$, these moments will actually be dominated by the rare samples of length $L$ which are already delocalized at $T$, i.e. the samples having $T_{c}(i, L)<T$. Since our numerical results indicate that the distribution of the pseudo-critical temperature $T_{c}(i, L)$ is a Gaussian with mean and width given respectively by Eqs (40) and (41), we obtain that the fraction of delocalized samples presents the following exponential decay in $L$

$$
\operatorname{Prob}\left[T_{c}(i, L)<T\right] \sim e^{-\left(T_{c}^{\infty}-T\right)^{2} L}
$$

This measure of the rare delocalized samples will govern the decay of the averaged loop distribution, and the correlation length defined in (51) thus involves the exponent $\nu=2$

$$
\xi(T) \underset{T \rightarrow T_{c}^{-}}{\longrightarrow}\left(T_{c}-T\right)^{-\nu} \text { with } \nu=2
$$

in contrast with the typical correlation length (50).

To better understand the emergence of two different correlation lengths, we have numerically measured the distribution over the samples $(i)$ of the free-energy $f^{(i)}(L, T)$ defined in Eq. (44). We obtain that for $T<T_{c}$

$$
f_{L}^{(i)}(T)=f(T)+\frac{a_{T}}{L}+\frac{\sigma_{T} u_{i}}{\sqrt{L}}
$$

where $a_{T}$ is temperature dependent and $u_{i}$ is a Gaussian random variable of zero mean and of variance 1

$$
G(u)=\frac{1}{\sqrt{2 \pi}} e^{-\frac{u^{2}}{2}}
$$

The averaged end-to-end loop distribution then reads 45

$$
\overline{P_{L}^{(i)}(L, T)}=\frac{1}{L^{c}} \overline{e^{L \beta f^{(i)}(L, T)}}=\frac{1}{L^{c}} e^{L \beta f(T)} \int_{-\infty}^{+\infty} d u G(u) e^{\sqrt{L} \beta \sigma_{T} u}=\frac{1}{L^{c}} e^{L \beta f(T)+L \frac{\beta^{2} \sigma_{T}^{2}}{2}}
$$


The difference between the correlation length $\xi(T)$ (51) and the typical correlation length (48) is due to the variance $\sigma_{T}^{2}$

$$
\frac{1}{\xi(T)}=\frac{1}{\tilde{\xi}(T)}-\frac{\beta^{2} \sigma_{T}^{2}}{2}
$$

In particular, to obtain the scaling $\frac{1}{\xi(T)} \sim\left(T_{c}-T\right)^{2}$ different from $\frac{1}{\xi(T)} \sim\left(T_{c}-T\right)$, the variance term in $\sigma_{T}^{2}$ has to cancel exactly the leading order in $\left(T_{c}-T\right)$ on the left hand-side.

So the picture that emerges of the present analysis is very reminiscent of what happens at strong disorder fixed points [33, 34] : the exponents $\tilde{\nu}=1$ and $\nu=2$ govern respectively the decay of typical/averaged loop distribution. Our conclusion is thus that the exponent $\tilde{\nu}=1$ governs the free-energy (49) that corresponds to a Lyapunov exponent, i.e. it describes the critical behavior of any typical sample, whereas the exponent $\nu=2=2 / d$ is the finite-size scaling exponent of Chayes et al 2 and is related to the variance of the distribution of pseudo-critical temperatures. Figure 8 of our previous paper 25] may be now interpreted as follows : for each sample, the critical region has a width of order $1 / L$, whereas the contact density averaged over the samples decay on a much wider scale $1 / \sqrt{L}$ that represents the sample-to-sample fluctuations of the pseudo-critical temperatures $T_{c}(i, L)$.

\section{SUMMARY AND DISCUSSION}

In this paper, we have used the recent progresses in the theory of finite-size scaling in disordered systems $\underline{3}, \underline{4}, \underline{5}$, [6, 7, [8] to study the role of disorder in Poland-Scheraga models. We have obtained that the numerical measure of the distribution of pseudo-critical temperature $T_{c}(i, L)$ over the samples is a very powerful tool to elucidate the true nature of random critical points. The comparison between the averaged shift $T_{c}^{a v}(L)-T_{c}(\infty)$ and the width $\Delta T_{c}(L)$ clarifies the role of disorder, and allows one to understand the non self averaging properties of various observables at $T_{c}(\infty)$ whenever disorder is relevant.

For $c=1.75$ corresponding to a second order transition with relevant disorder, we have obtained that both the width $\Delta T_{c}(L)$ and the shift $\left[T_{c}(\infty)-T_{c}^{a v}(L)\right]$ decay as $L^{-1 / \nu_{\text {random }}}$ with the same new exponent $\nu_{\text {random }} \sim 2.7>2>\nu_{\text {pure }}$.

For $c=1.5$ corresponding to a second order transition with marginal disorder, we have obtained that the exponent is unchanged $\nu=2$ with respect to the pure case, but that disorder is nevertheless relevant : the width $\Delta T_{c}(L) \sim L^{-1 / 2}$ is of the same order of the shift $T_{c}^{a v}(L)-T_{c}(\infty) \sim L^{-1 / 2}$ and this means that thermodynamic observables (apart from the free-energy) remain distributed at criticality. We have checked it numerically for the contact density.

For $c=2.15$ corresponding to a first order transition in the pure case, we have obtained that the width $\Delta T_{c}(L) \sim$ $L^{-1 / 2}$ dominates over the shift $T_{c}^{a v}(L)-T_{c}(\infty) \sim L^{-1}$. The presence of two correlation length exponents $\nu=2$ and $\tilde{\nu}=1$ is reminiscent of what happens at strong disorder fixed points [33, 34], and we have explained how these two exponents appear respectively in the typical and averaged loop distributions.

\section{Acknowledgments}

It is a pleasure to thank A. Billoire, G. Giacomin, J. Houdayer, F. Iglói and J. Jacobsen for discussions.

[1] A.B. Harris, J. Phys. C 7, 1671 (1974).

[2] J.T. Chayes, L. Chayes, D.S. Fisher and T. Spencer, Phys. Rev. Lett. 57, 2999 (1986); R.R.P. Singh and M.E. Fisher, ibid., 60, 548 (1988).

[3] S. Wiseman and E. Domany, Phys Rev E 52, 3469 (1995).

[4] A. Aharony, A.B. Harris, Phys Rev Lett 77, 3700 (1996).

[5] F. Pázmándi, R.T. Scalettar and G.T. Zimányi, Phys. Rev. Lett. 79, 5130 (1997).

[6] S. Wiseman and E. Domany, Phys. Rev. Lett. 81, 22 (1998) ; Phys Rev E 58, 2938 (1998).

[7] A. Aharony, A.B. Harris and S. Wiseman, Phys. Rev. Lett. 81, 252 (1998).

[8] K. Bernardet, F. Pázmándi and G.G. Batrouni, Phys. Rev. Lett. 80, 4477 (2000).

[9] D. Poland and H.A. Scheraga eds., Theory of Helix-Coil transition in Biopolymers, Academic Press, New York (1970).

[10] G. Forgacs, J.M. Luck, Th.M. Nieuwenhuizen and H. Orland, Phys. Rev. Lett., 57, 2184 (1986); J. Stat. Phys., 51, 29 (1988).

[11] B. Derrida, V. Hakim and J. Vannimenus, J. Stat. Phys., 66, 1189 (1992).

[12] S.M. Bhattacharjee and S. Mukherjee, Phys. Rev. Lett., 70, 49 (1993); Phys. Rev. E48, 3483 (1993). 
[13] H. Kallabis and M. Lässig, Phys. Rev. Lett., 75, 1578 (1995).

[14] D. Cule and T. Hwa, Phys. Rev. Lett., 79, 2375 (1997).

[15] L-h Tang and H. Chaté, Phys. Rev. Lett., 86, 830 (2001).

[16] T. Garel and C. Monthus, Eur. Phys. J. B, 46, 117 (2005).

[17] Y. Imry and M. Wortis, Phys. Rev. B, 19, 3580 (1979)

[18] M. Aizenman and J, Wehr, Phys. Rev, Lett., 62, 2503 (1989); (E) 64, 1311 (1990)

[19] K. Hui and A.N. Berker, Phys. Rev, Lett., 62, 2507 (1989)

[20] J. Cardy, Physica A, 263, 215 (1999)

[21] J. Cardy and J. L. Jacobsen, Phys. Rev. Lett., 79, 4063 (1997); Nucl. Phys. B, 515701 (1998); J. L. Jacobsen, Phys. Rev. E, 61, R6060 (2000).

[22] J-Ch. Anglès d'Auriac and F Iglói, Phys. Rev.Lett. 90, 190601 (2003).

[23] M.T. Mercaldo, J-Ch. Anglès d'Auriac and F Iglói, Phys. Rev. E 69, 056112 (2004).

[24] M.T. Mercaldo, J-Ch. Anglès d'Auriac and F Iglói, Europhys. Lett. 70, 733 (2005).

[25] T. Garel and C. Monthus, J. Stat. Phys: Theory and Experiment, P06004 (2005).

[26] M.S. Causo, B. Coluzzi and P. Grassberger Phys. Rev. E 62, 3958 (2000)

[27] E. Carlon, E. Orlandini and A.L. Stella, Phys. Rev. Lett., 88, 198101 (2002).

[28] M. Baiesi, E. Carlon, and A.L. Stella, Phys. Rev. E, 66, 021804 (2002); M. Baiesi, E. Carlon, Y. Kafri, D. Mukamel, E. Orlandini and A.L. Stella, Phys. Rev. E, 67, 021911 (2002).

[29] Y. Kafri, D. Mukamel and L. Peliti, Phys. Rev. Lett., 85, 4988 (2000); Y. Kafri, D. Mukamel and L. Peliti, Eur. Phys. J. B, 27, 135 (2002).

[30] B. Coluzzi, cond-mat/0504080

[31] G. Giacomin and F.L. Toninelli, math.PR/0506431

[32] M. Fixman and J.J. Freire, Biopolymers, 16, 2693 (1977)

[33] D. Fisher, Phys. Rev. B 516411 (1995).

[34] F. Iglói and C. Monthus, Phys. Rep. 412, 277 (2005). 